\title{
Characteristics of community-acquired pneumonia in hospitalized lebanese children and their correlation
}

\begin{abstract}
Aim: To study the radiological findings of community-acquired pneumonia in correlation with clinical signs and symptoms, as well as laboratory findings in children and adolescents hospitalized.

Material and methods: We retrospectively reviewed the medical records of children who were hospitalized for community-acquired pneumonia. A descriptive study was followed by a correlation study of patients' clinical signs and symptoms, physical examination, and laboratory studies, with the patients' radiologic finding.

Results: We reviewed the medical records of 268 pediatric patients who met criteria The most common symptoms were cough $(94.4 \%)$ and fever $(89.9 \%)$. Crackles $(36.9 \%)$ heard and focal infiltrate $(37.7 \%)$ were the most common findings on physical exam and radiologic imaging, respectively. Among the laboratory studies performed, only C-reactive protein was found to be elevated with prolonged duration of symptoms $(\mathrm{p}<0.05)$. With the exception of abdominal pain $(\mathrm{p}=0.009)$, there were no significant differences relating patient symptoms with radiologic findings.

Conclusion: In this study, no clinical or laboratory finding could be sufficient for the diagnosis and therapeutic decision as found in previous studies. Newer laboratory tests and etiologic search methods should be made available, until then, a high clinical index of suspicion is useful in our developing country.
\end{abstract}

Keywords: community-acquired-pneumonia, clinical findings, laboratory results, radiologic features
Volume 6 Issue 4 - 2017

\author{
Ralph R Kamel,' Raymond E Kamel,'² Dany A \\ Curi, ${ }^{3}$ Dany A Al Hamod ${ }^{4}$ \\ 'Hotel Dieu de France Hospital University Medical Center, Saint \\ oseph University, Lebanon, \\ 2Department of Pediatrics, Saint George Hospital University \\ Medical Center, University of Balamand, Lebanon \\ ${ }^{3}$ Department of Pediatrics, Division of Hematology and \\ Oncology, Saint George Hospital University Medical Center \\ University of Balamand, Lebanon \\ ${ }^{4}$ Department of Pediatrics, Division of Neonatology, Saint \\ George Hospital University Medical Center, University of \\ Balamand, Lebanon
}

\begin{abstract}
Correspondence: Dany A. Al Hamod, MD, Department of Pediatrics, Division of Neonatology, Saint George Hospital University Medical Center, University of Balamand, Beirut, Lebanon,Tel 96|3683209, Email dany_alhamod@hotmail.com
\end{abstract}

Received: February 25, 2017 | Published: March 27, 2017

\section{Introduction}

One of the most prevalent and potentially lethal illnesses is community-acquired pneumonia (CAP). About half of affected children worldwide receive appropriate medical care. ${ }^{1,2}$ Even though children in developing countries suffer the highest burden of the disease, ${ }^{3-5}$ children in developed countries continue to face high disease burden despite better nourishment and immunizations. ${ }^{6}$

The era of infectious vaccination and antimicrobial therapy has led to a decline in the incidence of infections and mortality related to community-acquired pneumonia. ${ }^{7,8}$ However, despite all efforts, it remains one of the leading causes of morbidity and mortality in children and accounts for a good proportion of hospitalizations. ${ }^{2,9}$ The frequent use of antibiotics has led to increasing antibiotic resistance among bacterial infections. This underlines the importance of an accurate diagnosis and knowledge of the etiologic agent responsible for developing pneumonia.

Despite CAP's widespread impact, childhood data on causative infectious organisms are few, mainly due to the difficulty of establishing the underline primary etiology. ${ }^{7}$ There are no definitive findings in a patient's medical history, physical examination, or chest radiograph that can categorically differentiate bacterial from viral or atypical pneumonia. ${ }^{9}$ Therefore, we sought to analyze for a correlation between the clinical history, physical exam findings, and radiological features characteristics of community acquired pneumonia at a university hospital in Lebanon. Therefore, we sought to analyze for a correlation between our patients' clinical signs and symptoms, physical examination, and laboratory studies, with the patients' radiologic finding.

\section{Material and methods}

\section{Study design}

This is a single-centered retrospective study involving children hospitalized for CAP at Saint Georges Hospital-University Medical Center, Beirut, Lebanon between January 2004 and December 2008. The study was approved by the hospital's institutional review board. Patient selection was based on the discharge diagnoses divided according to the ICD-9 code: viral, pneumococcal, bronchopneumonia (organism unspecified), pneumonia (organism unspecified), acute bronchitis and viral illness. Excluded from the study were patients with the diagnoses of foreign body aspiration, drowning, chronic neurologic and respiratory illnesses, cardiac anomalies, and the patients hospitalized in the last 15 days before their admission. To note, patients treated for asthma, hyperreactive airways, or previous episodes of bronchiolitis were not excluded from this study.

\section{Data collection and snalysis}

Data was obtained from the patients' medical records and involved epidemiologic information, clinical information, pertinent physical exam findings, paraclinical information, treatment, and complications were collected.

Data collection and descriptive analysis were performed on SPSS software (19th version). We used the statistical technique ANOVA to compare the mean white blood cell (WBC) count, absolute neutrophil count (ANC) and C-reactive protein (CRP) level with different radiographic studies. Mean values considered statistically different had a p-value $<0.05$. 
Significant difference between groups was identified by a multiple comparison test (Bonferroni test). Xi-square or fisher exact test were used to compare the symptoms and physical findings among patients with the following radiologic findings: focal consolidation, focal infiltrates, diffuse infiltrates or normal chest X-ray (CXR). In addition, the fisher's exact test was used to correlate clinical signs and symptoms with blood culture results.

\section{Results}

\section{Patient characteristics}

We reviewed the medical records of 268 patients who met criteria. The study included $53.0 \%$ males and $47.0 \%$ females. The majority of patients were under 24 months (38.4\%) and between 25 to 60 months (42.2\%) of age. Only $19.4 \%$ of patients were over the age of 61 months. The 3months throughout the year that accounted for highest number of admissions were the winter months of January (16.4\%), February $(13.0 \%)$ and December (11.9\%). The period from the beginning of October till the end of March (autumn-winter months) accounted for $61.3 \%$. About one-third (31.7\%) of the patients were treated with antibiotics prior to their admission.

\section{Medical history}

After collecting and analyzing each patient's medical history, we then categorized pertinent information into 4 categories: No pertinent past medical history $(77.2 \%)$, hyperreactive airways (14.9\%), frequent otitis media (OM) or recurrent upper respiratory tract infection (URTI) (7.8\%), and premature birth (14.2\%).

\section{Symptoms}

Figure 1 lists the symptoms of the 268 patients included in this report. The most common symptoms reported were cough $(94.4 \%)$ and fever $(89.9 \%)$. What was noted and not mentioned in this figure that the patients had good general condition in $47.4 \%$ of the cases, $4.9 \%$ were just ill looking, $3.7 \%$ were irritable, $6.7 \%$ were lethargic, $13.8 \%$ had variable degrees of dehydration, and respiratory distress was present in $20.1 \%$.

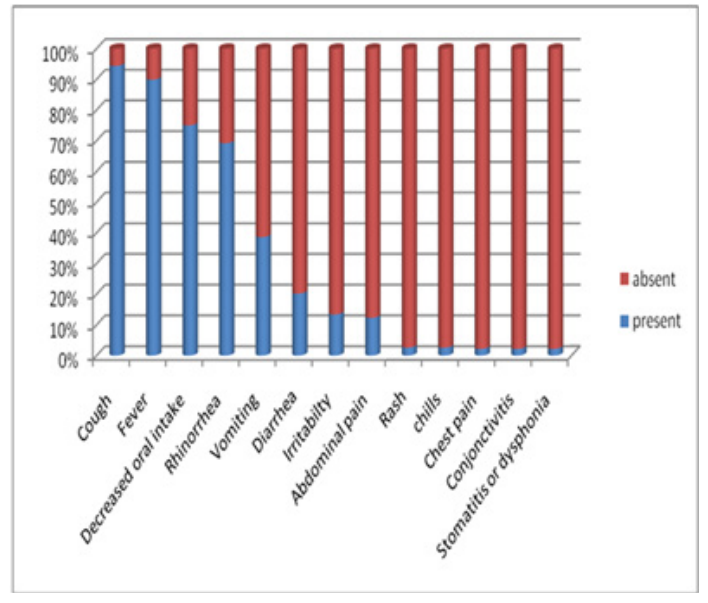

Figure I Recorded symptoms of community acquired pneumonia.

\section{Physical exam findings}

The most common clinical respiratory examination findings on admission were crackles (36.9\%), decreased air entry $(29.1 \%)$, and rhonchi $(9.7 \%)$. The resident who first evaluated each patient mentioned tachypnea and concomitant $\mathrm{OM}$ in $25 \%$ and $9.7 \%$ of the cases respectively. The only significant variables related to age were the presence of decreased air entry that was noticed to be more prevalent among children older than 2 years of age $(p=0.025)$ and the rhonchi that were noted among children under 5years of age $(\mathrm{p}=0.003)$.

\section{Radiology results}

Chest X-rays were performed during the first 24hours of hospitalization. Figure 2 shows the results classified into 4 categories: Presence of a focal consolidation (37.7\%), focal infiltrates $(25.7 \%)$, diffuse infiltrates (17.2\%), and absence of these findings (19.4\%). Of note, pleural effusion was found in $5.6 \%$ of the cases. Sex, age and the presence of fever were not statistically significant with respect to the presence of radiologic findings in this study.

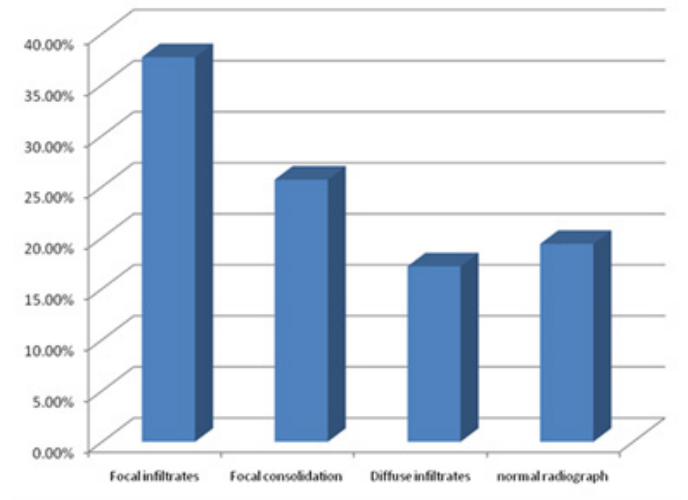

Figure 2 Radiologic findings of patients hospitalized for pneumonia.

\section{Laboratory results}

A CBC was performed on 267 patients. The mean WBC count was $15784 / \mathrm{mm} 3$. CBC differential was available for 266 patients and showed a mean ANC of 10886/mm3. CRP was measured on 215 patients $(80.2 \%)$; it had a mean of $10.7 \mathrm{mg} / \mathrm{dl}$.CRP increased with increasing duration of symptoms $(p<0.05)$. Blood cultures were performed in 173patients, and was positive in 9 cases; one case of Group A $\beta$ hemolytic Streptococcus, 4 cases of Streptococcus pneumonia and 4 cases of Staphylococcus epidermidis considered as contamination.

Pleural fluid was cultured in 6 cases, and was positive in one case that grew staphylococcus aureus. Cold agglutinin was tested in 5.2\% of cases on admission, and no repeated serologies were included in the patients' medical records. Two patients tested positive. Mycoplasma IgM antibodies were searched for by enzyme-linked immunosorbent assay (ELISA) in $7.0 \%$ of the cases and only two cases were positive that were different from the cases with positive cold agglutinin test. Four cases tested positive for respiratory syncytial virus (RSV) bvpolymerase chain reaction (PCR) from nasal aspiration.

\section{Etiologic agents}

Etiology remained unknown in $93.7 \%$ of the cases. A bacterial agent was found by blood culture, pleural fluid culture or pleural fluid PCR in 7 cases (2.6\%), and RSV was found in 4 cases of which one had radiographic findings of focal consolidation and pleural effusion with clinical symptoms lasting more than 5 days with recent deterioration. Mycoplasma pneumonia was suspected in 4 cases.

\section{Complication and video-assisted thoracoscopic surgery (VATS)}

Pleural effusion was seen in $5.6 \%$ of the cases and empyemas in 8 cases $(3 \%)$ where VATS or chest tube insertion were necessary. Lung abscess was not seen in any case. 


\section{Correlation results}

Correlation of clinical and laboratory findings with presence of consolidation: All symptoms showed no significant difference related to the radiographic findings except for abdominal pain; $23 \%$ of patients with consolidation had abdominal pain and $48.5 \%$ of the patients with abdominal pain had focal consolidation while only $6 \%$ of the patients who had abdominal pain had normal CXR. Xi square test showed significant $\mathrm{p}$ value $(\mathrm{p}=0.009)$. Table 1 represents the prevalence of clinical findings in relation to different radiographic features. Table 2 shows the physical findings with the radiologic features.

We also looked at the WBC count, $\mathrm{ANC}$ and CRP in relation to the radiographic features. Table 3 shows these results. When the $p$ value of the analysis of variance (ANOVA) is less than 0.05 , two or more groups have statistically different means. Therefore, a multiple comparison test was performed using the Bonferroni method (Table $4)$.

Correlation of clinical and laboratory findings with positive blood cultures: Table 5 illustrates the clinical symptoms in correlation with blood cultures. Results show no correlation of clinical signs and symptoms with the known positive bacterial etiology. Table 6 studies the clinical exam findings of crackles and decreased air entry with the streptococcal infection.

Table I Radiologic findings in relation with patient(s) symptoms

\begin{tabular}{|c|c|c|c|c|c|}
\hline & \multicolumn{5}{|c|}{ Radiologic Findings } \\
\hline & & & & & $\chi^{2}$ \\
\hline & Normal CXR & Focal Consolidation & Focal Infiltrates & Diffuse Infiltrates & P value \\
\hline & $\mathrm{N}=52$ & $\mathrm{~N}=69$ & $N=10 \mid$ & $\mathrm{N}=45$ & \\
\hline \multirow[t]{2}{*}{ Cough } & 50 & 67 & 92 & 43 & $0.327 \mathrm{a}$ \\
\hline & $-96.20 \%$ & $-97.10 \%$ & $-91.10 \%$ & $-95.60 \%$ & \\
\hline \multirow[t]{2}{*}{ Rhinorrhea } & 37 & 39 & 57 & 23 & 0.202 \\
\hline & $-71.20 \%$ & $-58.20 \%$ & $-56.40 \%$ & $-51.10 \%$ & \\
\hline \multirow[t]{2}{*}{ Diarrhea } & 8 & 14 & 19 & 13 & 0.396 \\
\hline & $-15.40 \%$ & $-20.30 \%$ & $-18.80 \%$ & $-28.90 \%$ & \\
\hline \multirow[t]{2}{*}{ Vomiting } & 25 & 23 & 39 & 16 & 0.399 \\
\hline & $-48.10 \%$ & $-33.30 \%$ & $-38.60 \%$ & $-35.60 \%$ & \\
\hline \multirow[t]{2}{*}{ Conjunctivitis } & 2 & 0 & 3 & 1 & $0.488 \mathrm{a}$ \\
\hline & $-3.80 \%$ & $0.00 \%$ & $-3.00 \%$ & $-2.20 \%$ & \\
\hline \multirow[t]{2}{*}{ Abdominal pain } & 2 & 16 & II & 4 & $0.009 *$ \\
\hline & $-3.80 \%$ & $-23.20 \%$ & $-10.90 \%$ & $-8.90 \%$ & \\
\hline \multirow[t]{2}{*}{ Decreased oral intake } & 39 & 48 & 77 & 35 & 0.727 \\
\hline & $-75.00 \%$ & $-69.60 \%$ & $-76.20 \%$ & $-77.80 \%$ & \\
\hline \multirow[t]{2}{*}{ Non pruritic rash } & 2 & 0 & 5 & 0 & $0.137 \mathrm{a}$ \\
\hline & $-3.80 \%$ & $0.00 \%$ & $-5.00 \%$ & $0.00 \%$ & \\
\hline \multirow[t]{2}{*}{ Stomatitis } & I & I & 3 & I & $0.926 a$ \\
\hline & $-1.90 \%$ & $-1.40 \%$ & $-3.00 \%$ & $-2.20 \%$ & \\
\hline \multirow[t]{2}{*}{ Irritability } & 7 & 5 & 15 & 9 & 0.251 \\
\hline & $-13.50 \%$ & $-7.20 \%$ & $-14.90 \%$ & $-20.00 \%$ & \\
\hline
\end{tabular}

Table 2 Radiologic findings in relation to physical exam findings

\begin{tabular}{|c|c|c|c|c|c|c|}
\hline & & \multicolumn{5}{|c|}{ Radiologic Findings } \\
\hline & & & & & & $\chi^{2}$ \\
\hline & & Normal CXR & Focal Consolidation & Focal Infiltrates & Diffuse Infiltrates & $P$ value \\
\hline \multirow{3}{*}{ Crackles } & & $\mathrm{N}=52$ & $\mathrm{~N}=69$ & $N=10 \mid$ & $\mathrm{N}=45$ & \\
\hline & No crackles & 33 & 39 & 65 & 31 & 0.536 \\
\hline & & $-63.40 \%$ & $-56.50 \%$ & $-64.30 \%$ & $-68.80 \%$ & \\
\hline \multirow{4}{*}{$\begin{array}{l}\text { Decreased air } \\
\text { entry }\end{array}$} & Presence of crackles & 19 & 30 & 36 & 14 & \\
\hline & & $-36.50 \%$ & $-43.50 \%$ & $-35.60 \%$ & $-45.10 \%$ & \\
\hline & yes & 11 & 31 & 23 & 13 & $0.008 *$ \\
\hline & & $-21.20 \%$ & $-44.90 \%$ & $-22.80 \%$ & $-28.90 \%$ & \\
\hline & No & 41 & 38 & 78 & 32 & \\
\hline \multirow{3}{*}{ Wheezing } & & $-78.80 \%$ & $-55.10 \%$ & $-77.20 \%$ & $-71.10 \%$ & \\
\hline & Yes & 41 & 66 & 81 & 38 & $0.026 *$ \\
\hline & & $-78.80 \%$ & $-95.60 \%$ & -80.2 & $-84.40 \%$ & \\
\hline \multirow{6}{*}{ Rhonchi } & No & 11 & 3 & 20 & 7 & \\
\hline & & $-21.20 \%$ & $-4.40 \%$ & $-19.80 \%$ & $-15.60 \%$ & \\
\hline & Yes & 44 & 65 & 91 & 41 & 0.365 \\
\hline & No & $-84.60 \%$ & $-94.20 \%$ & $-90.10 \%$ & $-91.10 \%$ & \\
\hline & & 8 & 4 & 10 & 4 & \\
\hline & & $-15.40 \%$ & $-5.80 \%$ & $-9.90 \%$ & $-8.90 \%$ & \\
\hline
\end{tabular}

*The test of independence (chi-square) has a $\mathrm{p}$ value statistically significant. 
Table 3 Laboratory results in correlation with radiological findings

\begin{tabular}{|c|c|c|c|c|c|}
\hline & & $\mathbf{N}$ & Mean & Std. Deviation & $\begin{array}{l}\text { ANOVA } \\
\text { p value }\end{array}$ \\
\hline \multirow[t]{5}{*}{ White Blood Cells } & Normal CXR & 52 & 14326 & 6695 & $0.002 *$ \\
\hline & Focal consolidation & 68 & 18720 & 9172 & \\
\hline & Focal infiltrates & 101 & 14200 & 7663 & \\
\hline & Diffuse infiltrates & 45 & 16280 & 7416 & \\
\hline & Total & 266 & 15732 & 8050 & \\
\hline \multirow[t]{5}{*}{ Neutrophil Count } & normal CXR & 52 & 9387 & 6527 & $0.000 I^{*}$ \\
\hline & Focal consolidation & 69 & 14252 & 9129 & \\
\hline & Focal infiltrates & 101 & 9239 & 7458 & \\
\hline & Diffuse infiltrates & 45 & 10564 & 6984 & \\
\hline & Total & 267 & 10786 & 7929 & \\
\hline \multirow[t]{5}{*}{ CRP mg/dl } & normal CXR & 44 & 6.37 & 10.55 & $0.000 I^{*}$ \\
\hline & Focal consolidation & 56 & 18.27 & 14.87 & \\
\hline & Focal infiltrates & 83 & 6.71 & 8.5 & \\
\hline & Diffuse infiltrates & 34 & 12.99 & $|4.4|$ & \\
\hline & Total & 217 & 10.61 & 12.78 & \\
\hline
\end{tabular}

Table 4 Bonferroni multiple comparison test

White Blood Cells
Normal CXR v/s Focal Consolidation $\mathrm{P}$
value $=0.016$
Focal infiltrates v/s Focal Consolidation
$P$ value $=0.002$
$P$ value $=0.002$

\section{Neutrophil count}

Normal CXR v/s Focal Consolidation $\mathrm{p}$ value $=0.004$

Focal infiltrates v/s Focal Consolidation $\mathrm{P}$ value $=0.000 \mathrm{I}$

\section{CRP mg/dl}

Normal CXR v/s Focal Consolidation $p$ value $=0.000 \mathrm{I}$

Focal infiltrates v/s Focal Consolidation $p$ value $=0.000 \mathrm{I}$

Focal infiltrates $v / s$ Diffuse infiltrates $p$ value $=0.05$

All other comparisons were not statistically significant.

Table 5 Clinical symptoms in correlation with a positive blood cultures S. pneumoniae

\begin{tabular}{|c|c|c|c|c|c|}
\hline & \multicolumn{5}{|c|}{ S.pneumoniaedetected } \\
\hline & \multirow{2}{*}{$\begin{array}{l}\text { No } \\
\mathbf{N}=\mathbf{2 6} \text { I }\end{array}$} & & \multicolumn{2}{|l|}{ Yes } & \multirow[b]{2}{*}{$p$ value } \\
\hline & & & $N=7$ & & \\
\hline \multirow[t]{2}{*}{ Abdominal pain } & 31 & & 2 & & $>0.05 \mathrm{~b}$ \\
\hline & $-11.90 \%$ & & $-28.60 \%$ & & \\
\hline \multirow[t]{2}{*}{ Decreased oral intake } & 193 & & 6 & & $>0.05 b$ \\
\hline & $-73.90 \%$ & & $-85.70 \%$ & & \\
\hline \multirow[t]{2}{*}{ Non pruritic rash } & 7 & & 0 & & $>0.05 \mathrm{~b}$ \\
\hline & $-2.70 \%$ & & $0.00 \%$ & & \\
\hline \multirow[t]{2}{*}{ Diarrhea } & 54 & & 0 & & $>0.05 \mathrm{~b}$ \\
\hline & $-20.70 \%$ & & $0.00 \%$ & & \\
\hline \multirow[t]{2}{*}{ Vomiting } & 101 & & 2 & & $>0.05 \mathrm{~b}$ \\
\hline & $-38.70 \%$ & & $-28.60 \%$ & & \\
\hline \multirow[t]{2}{*}{ Fever d } & 235 & & 7 & & $>0.05 \mathrm{~b}$ \\
\hline & $-90.00 \%$ & & $-100.00 \%$ & & \\
\hline \multirow[t]{2}{*}{ Cough } & 247 & & 6 & & $>0.05 \mathrm{~b}$ \\
\hline & $-94.60 \%$ & & $-85.70 \%$ & & \\
\hline \multirow[t]{2}{*}{ Rhinorrhea } & 152 & 5 & & $>0.05 \mathrm{~b}$ & \\
\hline & $-58.70 \%$ & $-71.40 \%$ & & & \\
\hline \multirow[t]{2}{*}{ irritability } & 36 & 0 & & $>0.05 \mathrm{~b}$ & \\
\hline & $-13.80 \%$ & $0.00 \%$ & & & \\
\hline White Blood Cells & 15694 & 16886 & & $>0.05 \mathrm{a}$ & \\
\hline Mean (standard deviation) & -8050 & -8011 & & & \\
\hline Neutrophil count & 10729 & 13078 & & $>0.05 \mathrm{a}$ & \\
\hline Mean (standard deviation) & -7896 & -8899 & & & \\
\hline CRP $\mathrm{mg} / \mathrm{dl}$ & 10 & 31 & & $<0.05 * a$ & \\
\hline Mean (standard deviation) & -12 & -17 & & & \\
\hline \multicolumn{6}{|l|}{ a independent samples $t$ test. } \\
\hline \multicolumn{6}{|c|}{ b Fisher exact test. } \\
\hline \multicolumn{6}{|c|}{ *Mean difference is statistically significant. } \\
\hline
\end{tabular}

Citation: Kamel RR, Kamel RE, Curi DA, et al. Characteristics of community-acquired pneumonia in hospitalized lebanese children and their correlation. J Pediatr Neonatal Care. 2017;6(4): I-6. DOI: I0.15406/jpnc.2017.06.00253 
Table 6 Difference between steacoustic findings in S. pneumonia

\begin{tabular}{|c|c|c|c|c|}
\hline \multirow[t]{8}{*}{ Crackles } & No crackles & 164 & 5 & c \\
\hline & & $-62.80 \%$ & $-71.40 \%$ & \\
\hline & Crackles on the right side & 35 & I & \\
\hline & & $-13.40 \%$ & $-14.30 \%$ & \\
\hline & Crackles on the left side & 33 & 0 & \\
\hline & & $-12.60 \%$ & $0.00 \%$ & \\
\hline & Crackles bilaterally & 29 & I & \\
\hline & & $-11.10 \%$ & $-14.30 \%$ & \\
\hline \multirow[t]{2}{*}{ Crackles } & No & 164 & 5 & $>0.05 b$ \\
\hline & Yes & 97 & 2 & \\
\hline \multirow[t]{10}{*}{ Decreased air entry } & Bilateral & 21 & 0 & c \\
\hline & & $-8 \%$ & $0.00 \%$ & \\
\hline & GBAE & 186 & 4 & \\
\hline & & $-71.30 \%$ & $-57.10 \%$ & \\
\hline & Left & 30 & I & \\
\hline & & $-11.50 \%$ & $-14.30 \%$ & \\
\hline & Right & 24 & 2 & \\
\hline & & $-9.20 \%$ & $-28.60 \%$ & \\
\hline & No & 186 & 4 & $>0.05 b$ \\
\hline & Yes & 75 & 3 & \\
\hline \multicolumn{5}{|l|}{ b Fisher exact test. } \\
\hline \multicolumn{5}{|c|}{ c no parametric statistical test is feasible because the conditions are not present. } \\
\hline \multicolumn{5}{|c|}{ *Mean difference is statistically significant. } \\
\hline
\end{tabular}

\section{Discussion}

The clinical signs and symptoms of this study were not related to the radiologic diagnosis of pneumonia. Abdominal pain was the only clinical sign that showed significant difference with the presence of lung consolidation. This result was seen in a study done by Bilkis et al. $(p=0.009),{ }^{10}$ where cough and chest pain were also correlated with pneumonia, however, patients younger than lyear of age or who were treated with "more than one bronchodilator treatment in the emergency unit" were excluded from the study. This can explain the variation in the results, as children younger than 12 months of age are more prone to having a viral respiratory infection specifically bronchiolitis than a pneumonia. ${ }^{11}$ Virkki et al. showed that nasal congestion was significantly more prevalent among RSV positive patients; our study didn't find such results, the difference in the study designs might be the cause. ${ }^{12}$

Among the physical exam findings, tachypnea, the most sensitive sign in several studies ${ }^{13,14}$ was not predominant in our study $(25 \%)$. This is most likely due to the lack of objective measurement and documentation. In addition, it was not adjusted to temperature and irritability. The symptom of tachypnea was more commonly seen in patients with wheezing. In contrary to Shah et al. ${ }^{15}$, who found tachypnea useful in distinguishing children with and without pneumonia only in patients without wheezing? Our study did not show the same results as it wasn't helpful in distinguishing pneumonia even in patients without wheezing. This is likely due to the differences in study design.

Decreased air entry was shown to be helpful clinical exam finding in detecting the presence of pneumonia, while crackles were not. Most of the literature has shown that both were significantly associated with pneumonia. A prospective cohort study demonstrated that patients with radiographic pneumonia were more likely than patients with normal chest radiographs to have among other clinical features crackles $(p=0.001)$ and decreased breath sounds $(p=0.034) .{ }^{11}$ Recall bias may be responsible for that divergence since crackles were considered absent if not mentioned to be present
Three laboratory findings were most frequently studied in correlation to radiographic pneumonia. WBC and ANC showed no difference when comparison between known and unknown etiology, based on blood culture, pleural culture and pleural PCR, was done. These results resemble those of previous studies showing that WBC and ANC are poor predictors of pneumonia's etiology. ${ }^{2,6,16} \mathrm{~A}$ study done by Korppi et al. showed no association between WBC and ANC with the radiographic alveolar versus interstitial infiltrates but it showed only significant difference in the evaluation of ANC and not WBC between bacterial pneumonia and viral pneumonia as well as mixed infections. ${ }^{17}$

CRP was significantly more prevalent in the consolidation group than all the three other radiological categories and it was shown to be more elevated in cases with positive blood culture, pleural culture or PCR with a significant $p$ value. The Korppi et al. prospective study showed the similar results where CRP was significantly higher in alveolar infiltrates versus interstitial infiltrates but was not variable among the different etiological categories. The sensitivity was too low $(26 \%$ when $C R P>60 \mathrm{mg} / \mathrm{l})$ in spite of a relatively acceptable specificity. ${ }^{15}$ On the contrary, a more recent meta-analysis concluded that serum CRP concentrations exceeding $40-60 \mathrm{mg} / \mathrm{L}$ weakly predict bacterial pneumonia in children with a positive predictive value of $64 \%{ }^{2}$

Furthermore, Cohen et al. recently published a study that evaluated the laboratory tests and their predictive value of rapid response to beta lactam antibiotics that they considered to be significant of bacterial etiology. They only found a significant correlation between procalcitonin and rapid response to beta lactam ant biotherapy. Among the limitations of that study is that the antibiotherapy was given empirically so the bacterial etiology wasn't objectively confirmed. ${ }^{18}$

\section{Conclusion}

Community acquired pneumonia is a very common diagnosis in children that, even though studied thoroughly, still presents major diagnostic, etiologic and therapeutic deficiencies. In this study, no 
clinical or laboratory findings could be sufficient for the diagnosis and therapeutic decision. In fact, decreased air entry reached a prevalence of $44.9 \%$ and elevated CRP could suggest a bacterial process, in contrast a low CRP couldn't rule it out. Our study has several limitations; it's a retrospective study, involving only hospitalized patients so it can't be extrapolated to every pneumonia case in the Lebanese population. There is still a considerable deficit in the etiologic diagnosis of community acquired pneumonia. Till now, there is no criterion standard for the diagnosis of pneumonia in children. ${ }^{19,20}$ The chest radiograph remains the diagnostic test of choice in tertiary care centers. ${ }^{11,21}$ Several studies tried to generate a clinical prediction algorithm for bacterial pneumonia ${ }^{11,19}$ but no one succeeded. ${ }^{5}$

More than $50 \%$ of cases remain without an etiologic diagnosis resulting in the unnecessary or inappropriate prescription of antibiotics ${ }^{22}$ and thus making the threat of antibiotic resistance closer.

A high clinical index of suspicion, following the WHO recommendation can be useful in our developing country until new laboratory tests viral and bacterial PCR, and specific bacterial serologies could be made more available.

\section{Acknowledgments}

None.

\section{Conflicts of interest}

Author declares there are no conflicts of interest.

\section{Funding}

None.

\section{References}

1. Wardlaw T, Johansson EW, Hodge M. Pneumonia: the forgotten killer of children. UNICEF/WHO. 2006.

2. Flood RG, Badik J, Aronoff SC. The utility of serum C-reactive protein in differentiating bacterial from nonbacterial pneumonia in children: a meta-analysis of 1230 children. Pediatr Infect Dis J. 2008;27(2): 95-99.

3. Thorn LK, Minamisava R, Nouer SS, et al. Pneumonia and poverty: a prospective population-based study among children in Brazil. BMC Infect Dis. 2011;11(1): 180.

4. Principi N, Esposito S. Management of severe community-acquired pneumonia of children in developing and developed countries. Thorax. $2011 ; 66(9): 815-822$.

5. Prayle A, Atkinson M, Smyth A. Pneumonia in the developed world. PaediatrRespir Rev. 2011;12(1):60-69

6. Krishne C, Thomson AH. Management of community-acquired pneumonia in children. Paediatr Drugs. 2007;9(6):401-411.

7. Nascimento-Carvalho CM, Ribeiro CT, Cardoso MR, et al. The role of respiratory viral infections among children hospitalized for communityacquired pneumonia in a developing country. Pediatr Infect Dis $J$. 2008;27(10):939-941.
8. Ranganathan SC, Sonnappa S. Pneumonia and other respiratory infections. Pediatr Clin North Am. 2009;56(1):135-156.

9. Heiskanen-Kosma T, Korppi M. Serum C-reactive protein cannot differentiate bacterial and viral aetiology of community-acquired pneumonia in children in primary healthcare settings. Scand J Infect Dis. 2000;32(4):399-402.

10. Bilkis M, Gorgal N, Carbone M, et al. Validation and Development of a Clinical Prediction Rule in Clinically Suspected Community-Acquired Pneumonia. PediatrEmer Care. 2010;26:399-405.

11. Lynch T, Platt R, Gouin S, et al. Can we predict which children with clinically suspected pneumonia will have the presence of focal infiltrates on chest radiographs?. Pediatrics. 2004;113(3 Pt 1):e186-189.

12. Virkki R, Juven T, Rikalainen H, et al. Differentiation of bacterial and viral pneumonia in children. 2002;57(5):438-441.

13. Palafox M, Guiscafré $H$, Reyes $H$, et al. Diagnostic value of tachypnoea in pneumonia defined radio logically. Arch Dis Child . 2000;82(1):41-45.

14. Mulholland EK, Simoes EA, Costales MO, et al. Standardized diagnosis of pneumonia in developing countries. Pediatr Infect Dis $J$. 1992;11(2):77-81.

15. Shah S, Bachur R, Kim D, et al. Lack of predictive value of tachypnea in the diagnosis of pneumonia in children. Pediatr Infect Dis $J$ 2010;29(5):406-409.

16. Heiskanen-Kosma T, Korppi M. Serum C-reactive protein cannot differentiate bacterial and viral aetiology of community-acquired pneumonia in children in primary healthcare settings. Scand J Infect Dis . 2000;32(4):399-402.

17. Korppi M, Heiskanen-Kosma T, Leinonen M. White blood cells, C-reactive protein and erythrocyte sedimentation rate in pneumococcal pneumonia in children. EurRespir J . 1997;10(5):1125-1129.

18. Cohen JF, Leis A, Lecarpentier T, et al. Procalcitonin predicts response to Beta-lactam treatment in hospitalized children with communityacquired pneumonia. PLoS One. 2012;7(5):e36927.

19. Bilkis M, Gorgal N, Carbone M, et al. Validation and Development of a Clinical Prediction Rule in Clinically Suspected Community-Acquired Pneumonia. Pediatr Emerg Care . 2010;26:399-405.

20. Hazir T, Nisar YB, Qazi SA, et al. Chest radiography in children aged 2-59 months diagnosed with non-severe pneumonia as defined by World Health Organization: descriptive multicentre study in Pakistan. BMJ. 2006;333(7569): 629 .

21. Neuman MI, Scully KJ, Kim D, et al. Physician assessment of the likelihood of pneumonia in a pediatric emergency department. Pediatr Emerg Care. 2010;26(11):817-822.

22. Marcos M.A. PhD, Torrres A. MD, PhD Viral Community-acquired Pneumonia Clinical Pulmonary Medicine. 18(2):60-64 